López-Mora, C., González Hernández, J., Garcés de los Fayos Ruiz, E.J. y Portoles Ariño, A. (2017). Versión reducida del cuestionario CMA de Metas para Adolescentes (CMA-R). Revista de Investigación Educativa, 35(2), 445-464 DOI: http://dx.doi.org/10.6018/rie.35.2.226461

\title{
Versión reducida del cuestionario CMA de Metas para Adolescentes (CMA-R)
}

\author{
Reduced version of the questionnaire for \\ goals adolescents (CMA-R)
}

\author{
Clara López-Mora*, Juan González Hernández**, \\ Enrique Javier Garcés de los Fayos Ruiz* y Alberto Portoles Ariño* \\ *Dto. Personalidad, Evaluación y Tratamiento psicológico. Facultad de psicología. Universidad de Murcia (España) \\ ** Dto. Personalidad, Evaluación y Tratamiento psicológico. Facultad de psicología. Universidad de Granada (España) \\ *** Dto. Personalidad, Evaluación y Tratamiento psicológico. Facultad de psicología. Universidad de Zaragoza (España)
}

\begin{abstract}
Resumen
La conducta del individuo se regula de acuerdo a unos objetivos que reflejan una concepción de sí mismo y del entorno. Conocer las motivaciones que desencadenan la conducta de los seres humanos en una dirección determinada, es sumamente interesante y más aún si somos capaces de determinar patrones y relaciones con el resto de características que componen a una persona. El objetivo del presente trabajo ha sido desarrollar una versión reducida del Cuestionario de Metas para Adolescentes (CMA; Sanz de Acedo Lizarraga, Ugarte, E Lumbreras, 2003) para su uso en la detección metas que persiguen los adolescentes. Utilizando el CFA se analiza la estructura dimensional de las metas en una muestra de 1385 participantes, 694 varones (51.1\%) y 691 mujeres $(49.9 \%)$, con una edad media de 15.13 años $(D T=1.88)$. Los resultados indican que tanto el CMA original como la versión reducida $(C M A-R)$ presentan un comportamiento psicométrico sólido y adecuado. Los niveles de consistencia interna para las distintas dimensiones de la versión original oscilan entre .875 y .664, y para la versión reducida entre .845 y .656 . Las correlaciones entre las dimensiones de ambas versiones son elevadas y estadísticamente significativas.

Palabras clave: identidad; adaptación; creencias psicosociales; personalidad.
\end{abstract}

Correspondencia: Dr. Juan González Hernández., jgonzalez@ugr.es, Dpto. Personalidad, Evaluación y Tratamiento Psicológico. Facultad de Psicología. Universidad de Granada (España). 


\begin{abstract}
The individual's behavior is regulated by objectives that reflect a conception of himself and the environment. Knowing the motivations that trigger the behavior of human beings in a particular direction is extremely interesting and even more so if we are able to determine patterns and relationships with the rest of the characteristics that make up a person. The aim of this study was to develop a smaller version of the Goals Questionnaire for Adolescents (CMA; Sanz de Acedo, Ugarte,ELumbreras, 2003) for use in detecting the adolescents' goals. Using CFA to analyze the three-dimensional structure of the Goals of a sample of 1385 members, $694 \mathrm{Men}(51.1 \%)$ and 691 women (49.9\%), mean age scammers analyzed 15.13 years (SD = 1.88). The results indicate that both the original version as reduced the CMA (CMA-R) have a solid and adequate psychometric properties. The levels of internal consistency for the various subscales of the original range between .875 and .664; for the short version .845 and .656 . The correlations between the subscales of both versions are high and statistically significant.

Key words: identity; adaptation; psychosocial beliefs; personality.
\end{abstract}

\title{
Introducción
}

Conocer cuáles son los motivos que movilizan a los seres humanos para ir avanzando en su vida individual y social es sumamente interesante, más aún si somos capaces de señalar la existencia y relaciones de patrones evolutivos marcados por la edad y los contextos sociales circundantes a la época que permitan entender, dar sentido y construir aquello que el ser humano desea alcanzar en las diferentes circunstancias de la vida, es decir, los objetivos que se marcan a corto y largo plazo, las metas.

Diversos autores subrayan la importancia de la construcción de metas y proyectos para la comprensión de la conducta presente(Monereo, 2007; Paoloni, Rinaudo, \& González-Fernández, 2011; Riveiro, Sánchez, Horcajo, \& Suárez, 2013; Zimmerman, 2008). La conducta del individuo se regula, en última instancia, de acuerdo con los proyectos que se construyen y que se reflejan en la concepción de sí mismo y del entorno en todos sus planos (religioso, sexual, político, vocacional) (Erikson, 1970; Gibson-Cline, 2000). Es importante reconocer que una persona no logra la identidad de una sola vez y en todos los planos al mismo tiempo, en este contexto desarrollarse viene a ser un proceso de transformación en lo que se desea ser (Broc, 2000; Grotevant, 1998; Ramos \& Delgado, 2004), y se constituye en un aspecto central del desarrollo humano.

Cuando se definen las metas se parte de que estos objetivos individuales de logro son representaciones internas de los estados deseados que se desean alcanzar (Austin \& Vancouver, 1996; Covington, 2000; Inglés, Martínez-Monteagudo, García-Fernández, Valle, \& Castejón, 2015).

La relación que subyace de la influencia de las características de personalidad, asociada a variables tales como motivación, inteligencia o la expresión emocional, a procesos de aprendizaje como la percepción de autoeficacia, etc., establecen además el carácter predictivo sobre aspectos como el rendimiento académico (Caprara, Barbaranelli, Pastorelli, \& Cervone, 2004; Pintrich \& Schunk, 2006), adherencia a aprendizajes de rendimiento (Brophy, 2005; Molina, Chorot, Valiente, \& Sandín, 2014), elecciones de vida (Ford \& Nicholls, 1992), entre otras. 
La comunidad científica comparte la afirmación de la adolescencia (Berger, 2007; Carretero, Palacios, \& Marchesi, 1985; Domínguez, 2008) como la etapa adecuada para formular metas, que no serán más que el fruto de los procesos psicosociales que se dan en ella, tales como la formación de la identidad (Kroger, 2004; Markus \& Kunda, 1986; Markus \& Nurius, 1986; Waterman, 1999), la consolidación de valores, las relaciones con los demás y la construcción de planes para el futuro, vocación etc. (Hechinger, 1992).

Es en la etapa adolescente donde la configuración de metas adquiere una importancia fundamental, ya que se trata de una etapa de construcción del ser humano (Cid, Espadalé, Carreras, \& Martínez, 2013; Krauskopf, 1982; Santillano, 2009; Viñas, González, García, Malo, \& Casas, 2015) y no como una etapa de transición (Zacarés, Iborra, Tomás, \& Serra, 2009). Este tratamiento conceptual plasma más coherentemente el desarrollo que se produce en la misma, integrándose lo social, lo psicológico y lo biológico, y permitiendo un alcance conceptual mayor, para definir y comprender la adolescencia y los componentes motivacionales.

De tal forma, el proceso de desarrollo en la adolescencia ha sido asociado en la literatura científica hacia patrones conductuales estereotipados como rebeldía, la inestabilidad o conflictividad (Casco \& Oliva, 2005; Liau, 2007), búsqueda de autonomía (Cid et al., 2013; Mayseless \& Scharf, 2007, Zacarés et al., 2009), egoísmo (Baker \& Palmer, 2006; Gardner, Roth, \& Brooks-Gunn, 2008), o comportamientos de riesgo (Freitas-Rosa, Gonçalves, \& Antunes, 2015; Ratcliff, Jenkins, Reiter-Purtill, Noll, \& Zeller, 2011). Las metas facilitan, además, la presencia de unas conductas u otras según la predominancia que el adolescente de a las diferentes metas existentes, provocando que su vida diaria se oriente de igual modo hacia actividades encaminadas a satisfacer o cumplir las metas planteadas, así como el tipo de metas que construirá vitalmente más adelante. De tal manera, el estilo de vida desarrollado por el adolescente está enormemente influenciado por la tipología de meta presente (Sanz de Acedo Lizarraga, Ugarte, \& Lumbreras, 2003).

Los modelos teóricos para examinar la motivación adolescente tanto de logro como social, basan sus argumentaciones tanto en la Teoría de Metas (Dweck, 1986; Elliot, Gable, \& Mapes, 2006; Nicholls, 1989) como en la Teoría de las Metas de Logro (Duda \& Nicholls, 1992; Guan, Xiang, McBride, \& Bruene, 2006). Ambos postulados coinciden en que las metas percibidas como fáciles o moderadamente difíciles, elegidas libremente o asignadas por uno mismo o por alguien externo con bastante credibilidad (o significatividad) para el adolescente y asociadas a un beneficio personal, son las que se aceptan rápidamente y se relacionan con una buena realización (Armas \& Hernández, 2006).

Los tipos de metas que los jóvenes se plantean, además tienen especialmente que ver con el desarrollo de tareas propias de su periodo evolutivo en aspectos particulares (escolares, vitales, etc.) (Salmerón, Gutiérrez-Braojos, Salmerón-Vílchez, \& Rodríguez, 2011), influenciadas además en términos de su bienestar subjetivo, por las interpretaciones que otros realizan sobre su propia vida, su propia satisfacción individual y viceversa (Sanz de Acedo Lizarraga et al., 2003).

La conducta se considera intencional cuando está dirigida al cumplimiento de metas, considerado como un concepto motivacional cuyo poder se encuentra estrechamente asociado con las emociones (Zimmerman, 2008). Además, el proceso de construcción psicosociológica que comienza a hacerse consciente en el adolescente, le dirige esfuer- 
zos hacia la proyección de una autoimagen positiva ante los compañeros y hacia sí mismo, a exteriorizar necesidades de autonomía, de equilibrio, de riesgo o de destacar, hacia el deseo de adquirir y mantener cierto prestigio (Kinnebrew, Biswas, Sulcer, \& Taylor, 2013), búsqueda de reconocimiento, status económico y social dentro de la propia comunidad (Navas, Soriano, Holgado, \& López, 2009), de dar explicación a sus decisiones, percepciones de bienestar o desajuste personal, configuración y desarrollo de su autoconcepto, son elementos que modulan el desarrollo de la personalidad (Sanz de Acedo Lizarraga, Ugarte, Lumbreras, \& Sanz de Acedo Baquedano, 2006).

El conocimiento de las aspiraciones de los adolescentes, permite una mayor comprensión de sus actos y las conductas tipo que presentan, necesitando para ello herramientas que permiten acceder al conocimiento de estas metas de forma temprana de cara a organizar, regular, orientar y justificar conductas, explicar muchas de sus decisiones, etc. (Sanz de Acedo Lizarraga et al., 2003).

Carroll, Durkin, Hattie, \& Houghton (1997), proponen en su Goal Setting Questionaire (GSQ) ocho tipos de metas propias de los adolescentes, diferenciando entre: educativas, profesionales, interpersonales, autoimagen, reconocimiento social, enunciativas, riesgo y físicas. Estas metas organizan, regulan, orientan y justifican sus conductas, explican muchas de sus decisiones, contribuyen tanto a su bienestar como a su desajuste personal, influyen en el desarrollo de su autoconcepto (Sanz de Acedo Lizarraga et al., 2003; Wentzel, 1993; Wentzel \& Wigfield, 1998).

Señalar una autoimagen positiva ante los compañeros como una meta considerada por los adolescentes, se traduce en el deseo de adquirir y mantener cierto prestigio, status, renombre en su círculo de pares y comunidad. Conductas cada vez más generalizadas, con una sólida concepción de autonomía (cognitiva, afectiva, conductual y normativa) (Emler \& Reicher, 1995). El distanciamiento de los progenitores es otro de los grandes motores de los adolescentes que sienten la necesidad de tomar decisiones fuera del control de los adultos, en especial cuando tratan de elegir amigos, ropa, actividades de ocio y objetos de moda (Ford \& Nicholls, 1992). De la misma manera, algunos jóvenes tienen metas vinculadas con actividades ilegales reforzadas por los iguales (Blackburn, 1993) y propias del desarrollo cognitivo típico de la edad madurativa, por ejemplo el "mito de imbecilidad" (Elkind, 1967). De ahí que Carroll et al. (1997) hayan introducido en sus evaluaciones una escala de riesgo.

La adolescencia, por otro lado, supone en los jóvenes cambios físicos y aptitudinales relevantes que generan metas asociadas con el deseo de destacar en el deporte, pertenecer a equipos de élite, tener un cuerpo sano, estar en buena forma y disfrutar del deporte (Castillo, Balaguer, \& Duda, 2002; Duda \& Nicholls, 1992).

El Cuestionario de Metas Adolescentes (CMA; Sanz de Acedo Lizarraga et al., 2003), abarca constructos de instrumentos sobre las inquietudes en esta época de la vida (metas personales, sociales, académicas...), dando lugar a una primera versión validada de 79 ítems y 7 dimensiones: Reconocimiento social (RS), relativa a necesidad que tienen los jóvenes de mostrar su imagen y valía principalmente ante los iguales y, en consecuencia, de conseguir reputación; Interpersonal (I), relativa a la necesidades compartir con los pares ideas y sentimientos más íntimos de manera clara, sincera y en condiciones de igualdad; Deportiva (D), relativa al interés por el ejercicio físico y la competición deportiva; Emancipativa (E), relativa al afán de libertad, disfrute, riesgo 
y crítica a la sociedad; Educativa (ED), relativa a la percepción que los adolescentes tienen sobre el logro académico y a los objetivos que deberían llenar la vida escolar de los adolescentes; Sociopolítica (SP), relativa a la preocupación por la participación en los problemas sociales y políticos; y Compromiso personal (CP), relativa al responsabilidad que adquieren para con sus objetivos y su capacidad subjetiva de llevarlos termino si abandonar la meta ante contratiempos.

Los estudios de validez de constructo llevados a cabo en las dimensiones del CMA (Sanz de Acedo Lizarraga et al., 2003), encuentran una estructura subyacente de naturaleza multidimensional formada por tres factores de primer orden: Imagen social (RS y D), Independencia personal y social (E y SP), y Competencia académica y social (ED e I).

En líneas generales, el CMA muestra adecuadas propiedades psicométricas y se ha empleado en diversas temáticas relacionada con las diferencias de género (Delgado, Inglés, García-Fernández, Castejón, \& Valle, 2010), su relación con las estrategias motivacionales (González \& Portolés, 2014; Portolés, 2013; Valle et al., 2007), estrategias de afrontamiento y autorregulación (González \& Hernández, 2009), personalidad adolescente (Sanz de Acedo Lizarraga et al., 2006), autoestima (Martínez, Cabanach, \& Pérez, 2004), valores (Suárez, Delgado, \& Jiménez, 2011; Rijo, Moreno, Herráez, \& Medina, 2014) o rendimiento académico (Gaxiola, González, Contreras, \& Gaxiola, 2012; Romero, Lugo, \& Villa, 2013; Rueda, 2014).

En este sentido, dado que la finalidad última de estos autoinformes es la identificación de tendencias de metas en los adolescentes, se hace interesante la construcción de una versión reducida que pueda administrarse de forma más ágil, eficaz y no invasiva como herramienta de exploración.

El objetivo de la presente investigación ha sido desarrollar una versión reducida del CMA, de rápida administración en la práctica, sin que ello suponga una pérdida significativa de la fiabilidad y validez del test original. La estructura dimensional de las metas, evaluada con la versión reducida, se analizará mediante análisis factorial confirmatorio. Guiado además, por la hipótesis de mantener para esta versión una fiabilidad y validez equiparables a la versión original, así como su estructura dimensional.

La muestra está formada por 1385 adolescentes y adultos jóvenes españoles, seleccionados de forma incidental de diferentes centros de Educación Secundaria Obligatoria y Bachillerato. Del total de la muestra, 694 (50.1\%) fueron varones y 691 $(49.9 \%)$ mujeres, con una edad media de 15.12años ( $\mathrm{DT}=1.88)$; oscilando entre los 13 y los 17 años.

\section{Método}

\section{Participantes}

La muestra está formada por 1385 adolescentes y adultos jóvenes españoles, seleccionados de forma incidental de diferentes centros de Educación Secundaria Obligatoria y Bachillerato. Del total de la muestra, 694 (50.1\%) fueron varones y 691 (49.9\%) mujeres, con una edad media de 15.12 años (DT=1.88); oscilando entre los 13 y los 17 años. 


\section{Instrumento}

Metas para adolescentes. Se utiliza el Cuestionario de Metas para Adolescentes (CMA; Sanz de Acedo Lizarraga et al., 2003). Este cuestionario en su versión final consta de un total de 79 ítems, en formato de respuesta tipo Likert de 6 puntos para las dimensiones: Reconocimiento social (RS) ("ser líder de un grupo"), Interpersonal (I) ("ser sincero con los demás"), Deportiva (D) ("estar en buena forma física"), Emancipativa (E) ("tener libertad para tomar mis propias decisiones"), Educativa (ED) ("conseguir notas alta para poder acceder a la universidad"), Sociopolítica (SP) ("pertenecer a un partido político") y Compromiso personal (CP) ("trabajar con ilusión para conseguir las metas que deseo"). Para el proceso de creación y validación del cuestionario se utilizó como base el GSQ (Goal Setting Questionnaire de Carroll et al., 1997), compuesto por 57 ítems agrupados ocho factores de primer orden y tres de segundo orden. Este fue traducido al castellano por un equipo de expertos (dos psicólogos, un sociólogo, un metodólogo, un lingüista y dos educadores), tras el filtrado pertinente se cambió el formato del instrumento y se añadieron 81 ítems dando lugar a la versión A del CMA con 131 ítems agrupados en 9 escalas que fue administrada a tres muestras independientes hasta obtener la versión final de 79 ítems empleada en este trabajo. Se trata de un autoinforme que ha sido creado y utilizado para población adolescente (Sanz de Acedo Lizarraga et al., 2003), y cuyos resultados previos indican que la consistencia interna oscila entre .845 y .656 .

\section{Procedimiento}

La administración del cuestionario se llevó a cabo de forma colectiva, en grupos de 15 a 30 estudiantes, durante el horario académico y en un aula acondicionada para este fin. El estudio fue presentado a los participantes como una investigación sobre las diversas "cosas importantes para ellos", asegurándoles la confidencialidad de sus respuestas según protocolo ético establecido (Helsinki, 2000), así como del carácter voluntario de su participación. La administración del cuestionario se realizó en todo momento bajo la supervisión de un investigador y cuenta con el consentimiento informado de los tutores legales de los menores.

\section{Análisis de datos}

Se realiza un análisis descriptivo de las subescalas que conforman el cuestionario original y la versión reducida referido a la media, desviación típica, asimetría, curtosis y el rango de las puntuaciones; además, se estima la consistencia interna de las subescalas mediante el coeficiente alfa de Cronbach.

Para desarrollar la versión reducida del CMA han sido seleccionados los ítems en función de criterios sustantivo-teóricos y psicométricos. En este caso, se seleccionan aquellos ítems con índices de discriminación, coeficientes estandarizados y una proporción de varianza explicada elevada en sus subescalas correspondientes. Para desarrollar la versión reducida del CMA se han llevado a cabo diferentes análisis factoriales confirmatorios. El método de estimación es el de mínimos cuadrados ponderados a partir de la matriz de correlaciones policóricas, fijando a 1 la varianza. Asimismo, y 
dado que el número de ítems de las subescalas no es uniforme, se intenta equiparar su número reduciendo en mayor medida las subescalas con mayor número de ítems. En este sentido no se eliminó ningún ítem. Como criterio de validación interna de la versión reducida se calculan las correlaciones de ambas versiones, original y reducida.

Una vez desarrollada la versión reducida del CMA, se realizan diversos análisis factoriales confirmatorios para determinar qué modelo de metas presenta un mejor ajuste, utilizando como método de estimación el de máxima verosimilitud robusta (RML). La varianza de las variables latentes se fija a la unidad, no permitiéndose por tanto la correlación entre los términos de error. Para determinar la bondad de ajuste de los modelos se tiene en cuenta la solución factorial, los diferentes índices de bondad del ajuste, los índices de modificación, los coeficientes estandarizados, el porcentaje de varianza explicado y la significación estadística.

Siguiendo las directrices de Brown (2006) y Kline (2005), los índices de bondad de ajuste utilizados fueron los siguientes: el test chi-cuadrado, la ratio entre el chi-cuadrado y los grados de libertad ( $\chi 2 / \mathrm{df})$, el índice de ajuste comparativo (CFI), el índice de ajuste general (GFI), el error cuadrático medio de aproximación (RMSEA) (y su intervalo confidencial), la raíz del residuo estandarizado cuadrático medio (SRMR) y el criterio de información de Akaike (AIC). Los modelos contrastados fueron el original de 79 ítems y la versión reducida de 26items.

\section{Resultados}

\section{Estadísticos descriptivos y estudio de la consistencia interna de la versión original y reducida del CMA}

En las tablas 1 y 2 se muestran los estadísticos descriptivos de las dimensiones que componen el CMA original y su versión reducida: media, desviación típica, asimetría, curtosis y el rango de las puntuaciones, así como el coeficiente alfa de Cronbach. Las dimensiones presentaron valores de asimetría y curtosis dentro del rango de normalidad, a excepción de la sociopolítica en ambas versiones.

Tabla 1

Estadísticos descriptivos de las dimensiones del Cuestionario de Metas para Adolescentes versión original

\begin{tabular}{cccccccc}
\hline Dimensiones & No ítems & $\alpha$ & Media & DT & Asimetría & Curtosis & Rango \\
\hline R. Social & 14 & .875 & 2.825 & .964 & .531 & -.217 & 5 \\
Interpersonal & 14 & .854 & 4.830 & .752 & -.738 & .297 & 4 \\
Deportiva & 10 & .896 & 3.738 & 1.163 & -.063 & -.805 & 5 \\
Emancipativa & 13 & .786 & 3.596 & .835 & .044 & -.425 & 4 \\
Educativa & 11 & .839 & 4.475 & .873 & -.597 & .103 & 5 \\
Sociopolítica & 7 & .710 & 2.626 & .951 & .668 & .344 & 5 \\
Comp. personal & 10 & .664 & 3.903 & .757 & -.181 & .219 & 5 \\
Escala (CMA) & 79 & .928 & & & & & \\
\hline
\end{tabular}




\section{Desarrollo de la versión reducida del CMA (CMA-R)}

Para desarrollar la versión reducida del CMA se llevaron a cabo diferentes análisis factoriales confirmatorios a nivel de dimensiones. Los criterios para la selección de los ítems fueron tanto psicométricos (coeficientes estandarizados e índices de discriminación) como sustantivo-teóricos, respetando siempre las siete escalas originales.

Tabla 2

Estadísticos descriptivos de las escalas del Cuestionario Reducido de Metas para Adolescentes

\begin{tabular}{cccccccc}
\hline Dimensiones & No ítems & A & Media & DT & Asimetría & Curtosis & Rango \\
\hline R. Social & 3 & .683 & 3.604 & 1.256 & .376 & -.629 & 5 \\
Interpersonal & 4 & .755 & 4.807 & .964 & -.847 & .557 & 5 \\
Deportiva & 4 & .845 & 3.001 & 1.413 & .410 & -.857 & 5 \\
Emancipativa & 4 & .703 & 4.196 & 1.113 & -.328 & -.505 & 5 \\
Educativa & 4 & .707 & 4.485 & 1.042 & -.585 & -.082 & 5 \\
Sociopolítica & 3 & .656 & 1.956 & 1.120 & 1.390 & 1.563 & 5 \\
$\quad$ Comp. & 4 & .688 & 4.542 & 1.041 & -.549 & -.215 & 5 \\
personal & & & & & & & \\
Escala & 26 & .833 & & & & & \\
(CMA-R) & & & & & & &
\end{tabular}

El rango de los coeficientes estandarizados y la proporción de varianza explicada, así como los índices de bondad de ajuste de cada escala, una vez aplicados los criterios mencionados, se recogen en la tabla 3. Como puede observarse, la versión reducida del CMA (CMA-R) quedó formada por un total de 26 ítems. Los índices de consistencia interna oscilaron entre .845 y .656. Los pesos estandarizados fueron todos elevados, positivos y estadísticamente significativos $(p<.05)$, al igual que la proporción de varianza explicada. En líneas generales, los índices de bondad de ajuste para las escalas fueron adecuados, excepto en el factor sociopolítico que presenta un comportamiento anómalo en cuanto a la disparidad de respuesta (en la versión original presenta la misma anomalía), posiblemente debido al modo en que están enunciados los ítems (lenguaje poco cercano a la jerga adolescente) y alno ser una meta prioritaria en los adolescentes, las respuestas se realizan sin una conciencia de causa lo suficientemente interiorizada para ellos.

Las correlaciones entre las dimensiones la versión reducida (analizadas en primer lugar) y las correlaciones entre la versión original y reducida (en segundo), señalan que las dimensiones de la versión reducida muestran correlaciones entre ellas, excepto la sociopolítica con la interpersonal y compromiso personal. Las correlaciones entre las dimensiones de ambas versiones fueron asimismo estadísticamente significativas $(p<.01)$, oscilando entre .903 (ED) y .761 (SP). Se muestra además el modelo explicativo de relaciones entre las dimensiones de la versión reducida (figura 1). 


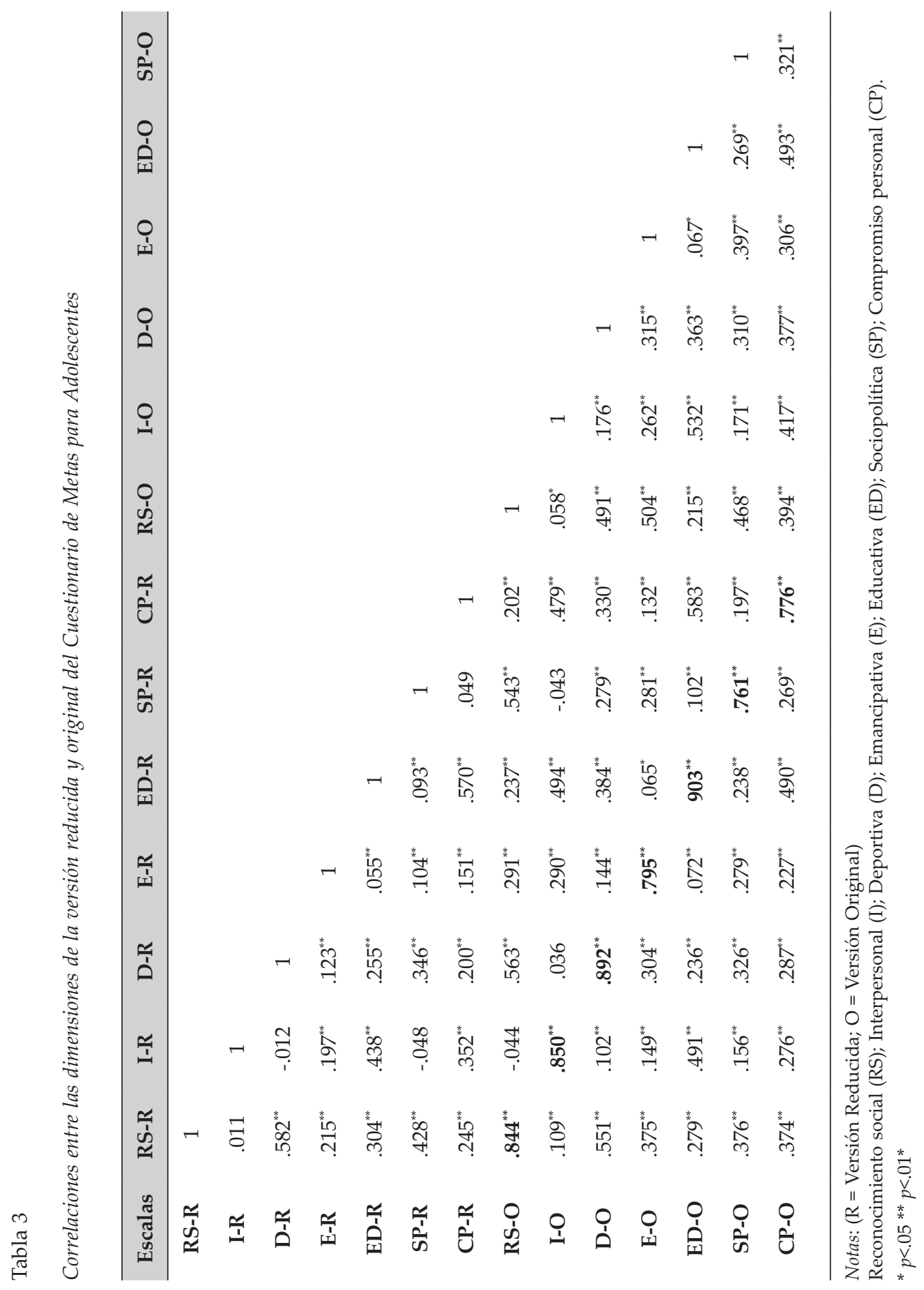




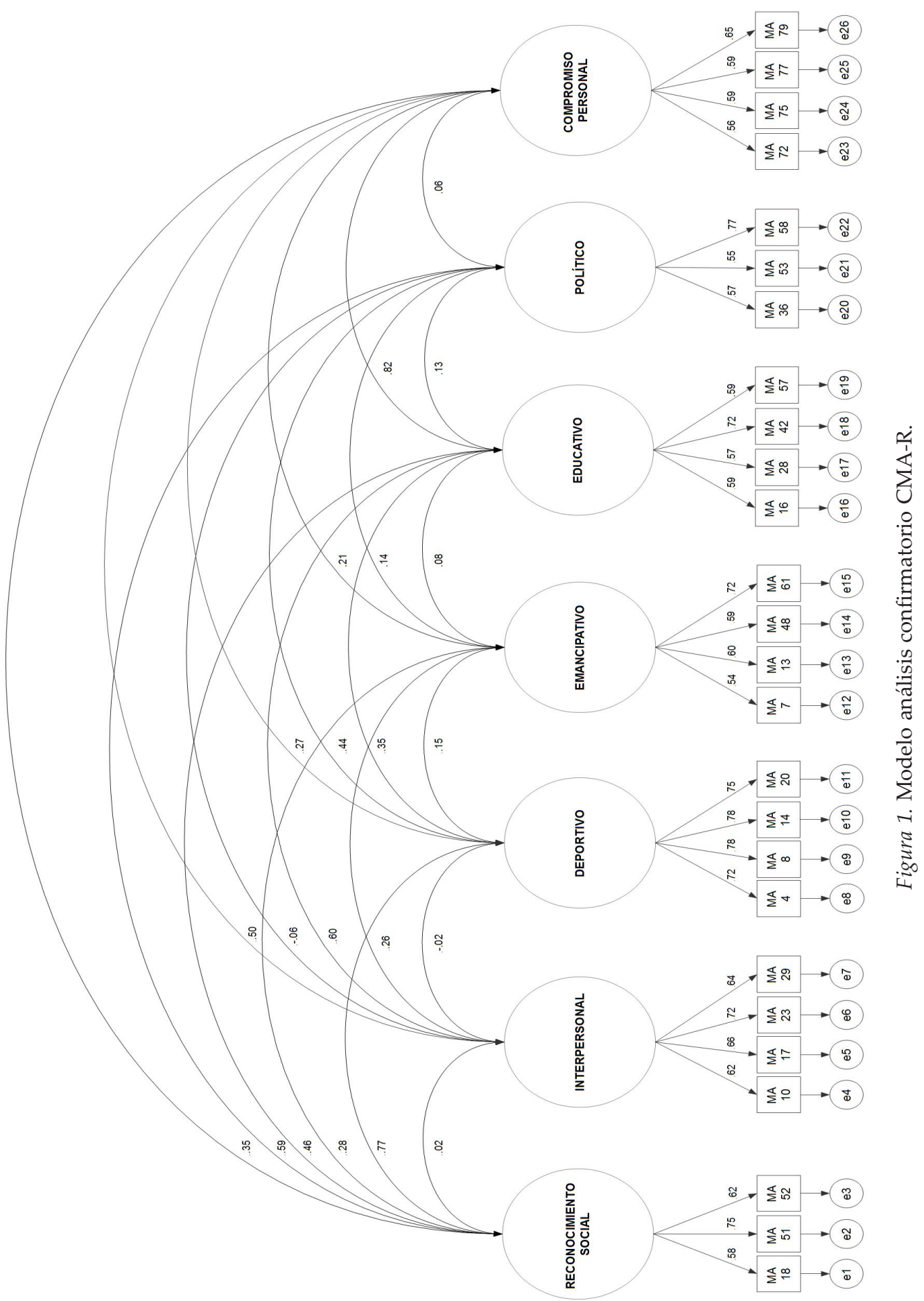




\section{Análisis factorial confirmatorio de la versión reducida del CMA}

Los índices de ajuste de los modelos propuestos se recogen en la tabla 4. Los resultados indican que el modelo B (versión reducida) fue el que mejores índices de ajuste presentó, en comparación con el A (versión original). El valor de chi-cuadrado fue adecuado, y dado que con muestras grandes es relativamente fácil obtener significación adecuada, se utilizó el ajuste por los grados de libertad ( $\chi 2 / \mathrm{df}$ ) obteniendo para ambas escalas ajuste adecuado. Los índices CFI, que indican un buen ajuste del modelo para valores próximos a 1 y se recomienda valores superiores a .90 obteniendo por tanto mejor índices para el modelo B o reducido. TLI compara el ajuste por grados de libertad del modelo propuesto y nulo (ausencia de relación entre variables), como se observa, el modelo B es más cercano a la unidad, por tanto presenta mejor ajuste. Del mismo modo os valores RMSEA y SRMR muestran un mejor ajuste para el modelo reducido. Todos los coeficientes estandarizados fueron estadísticamente significativos $(p<.05)$, oscilando entre .55 y .78 La proporción de varianza explicada osciló entre .765 y .575. La correlación entre las variables latentes también fue estadísticamente significativa, oscilando con valores situados entre .903 (ED) y .761 (SP). Los ítems que componen la versión abreviada del CMA se recogen en la tabla 5.

Tabla 4

Índices de bondad de ajuste para los modelos teóricos de metas

\begin{tabular}{cccccccc}
\hline Modelo & $\mathbf{X}^{2}$ & DF & $\chi^{2} / \mathbf{d f}$ & CFI & TLI & RMSEA & SRMR \\
\hline $\begin{array}{c}\text { A. } \\
\text { ORIGINAL }\end{array}$ & 13624.88 & 2981 & 4.571 & .718 & .708 & .051 & .0799 \\
\hline $\begin{array}{c}\text { B. } \\
\text { REDUCIDA }\end{array}$ & 1121.959 & 278 & 4.03 & .919 & .905 & .047 & .0473 \\
\hline
\end{tabular}

Notas: DF: Grados de libertad; GFI: índice de ajuste comparativo; GFI: índice de ajuste general; RMSEA: error cuadrático medio de aproximación; IC: Intervalo confidencial; SRMR: raíz del residuo estandarizado cuadrático medio; AIC: criterio de información de Akaike.

Tabla 5

Factores, $n^{-o}$ de ítem (NI), definición del ítem del CMA-R

\begin{tabular}{|c|c|c|c|c|}
\hline Factores & $\begin{array}{l}\text { № de } \\
\text { ítem }\end{array}$ & Descripción & V.O & V.R \\
\hline \multirow{3}{*}{$\begin{array}{l}\text { Reconocimiento } \\
\text { social }\end{array}$} & 02 & Ser considerado un héroe & $x$ & \\
\hline & 12 & Tener mucho poder & $x$ & \\
\hline & 18 & Ser líder de un grupo & $x$ & $x$ \\
\hline
\end{tabular}




\begin{tabular}{|c|c|c|c|}
\hline & 19 & Tener bastante dinero & $x$ \\
\hline & 25 & Alcanzar prestigio social & $x$ \\
\hline & 26 & Llevar los últimos diseños de moda & $x$ \\
\hline & 30 & Ser centro de atención de los demás & $x$ \\
\hline & 33 & Vestir de manera llamativa & $x$ \\
\hline & 34 & Obtener mejores notas que mis amigos & $x$ \\
\hline & 44 & Ser considerado superior a los demás & $x$ \\
\hline & 45 & Comprar siempre lo que quiero & $x$ \\
\hline & 50 & Llegar a ser gerente de una empresa & $x$ \\
\hline & 51 & Estar entre los mejores en todas las actividades & $x$ \\
\hline & 52 & Ser mencionado en situaciones especiales & $x$ \\
\hline Interpersonal & 6 & Ayudar a los demás & $x$ \\
\hline & 10 & Ser sincero con los demás & $x$ \\
\hline & 11 & $\begin{array}{l}\text { Respetar a la otra persona en una relación de } \\
\text { amistad }\end{array}$ & $x$ \\
\hline & 17 & Escuchar a los demás & $x$ \\
\hline & 23 & Ser justo con los demás & $x$ \\
\hline & 24 & Ser perdonado si ofendo a otras personas & $x$ \\
\hline & 29 & Ser leal con los demás & $x$ \\
\hline & 35 & Ser una persona de confianza & $X$ \\
\hline & 40 & Relacionarme con la gente & $x$ \\
\hline & 59 & Aceptar a los demás tal como son & $X$ \\
\hline & 66 & Expresar mis opiniones sin ofender a nadie & $x$ \\
\hline & 67 & $\begin{array}{l}\text { Identificarme con quienes me comunican sus emo- } \\
\text { ciones }\end{array}$ & $x$ \\
\hline & 68 & Reunirme con amigos & $x$ \\
\hline & 69 & Ser delicado en las conversaciones con los demás & $x$ \\
\hline Deportiva & 04 & Pertenecer a un equipo deportivo & $x$ \\
\hline & 08 & Sobresalir en deporte & $x$ \\
\hline & 14 & Jugar en equipos destacados & $X$ \\
\hline & 20 & Ser mejor que otros en deporte & $x$ \\
\hline & 27 & Estar en buena forma física & $x$ \\
\hline & 41 & Mejorar mis marcas deportivas & $x$ \\
\hline & 56 & Practicar el ejercicio físico & $x$ \\
\hline & 60 & Programar las actividades físicas & $x$ \\
\hline & 63 & Disfrutar con el deporte & $x$ \\
\hline & 65 & Relajarme con el deporte & $x$ \\
\hline
\end{tabular}




\begin{tabular}{|c|c|c|c|}
\hline Emancipativa & 03 & Divertirme en todo momento & $x$ \\
\hline & 07 & Hacer las cosas a mi manera & $X$ \\
\hline & 13 & Poder vivir por mi cuenta & $X$ \\
\hline & 31 & Asistir a muchas fiestas & $x$ \\
\hline & 32 & Realizar actividades de riesgo & $X$ \\
\hline & 37 & Realizar actividades distintas de las habituales & $x$ \\
\hline & 39 & Vivir alejado de la familia & $X$ \\
\hline & 48 & Tener libertad para tomar mis propias decisiones & $x$ \\
\hline & 49 & Satisfacer mis impulsos lo antes posible & $x$ \\
\hline & 55 & Hacer cosas prohibidas & $x$ \\
\hline & 61 & Tener libertad para hacer lo que yo quiera & $x$ \\
\hline & 62 & Rechazar lo que me imponen & $X$ \\
\hline & 64 & Disfrutar del sexo & $x$ \\
\hline Educativa & 01 & Ser buen estudiante & $x$ \\
\hline & 05 & Aprobar los exámenes & $X$ \\
\hline & 09 & Aprender cosas nuevas & $x$ \\
\hline & 16 & Finalizar las tareas en el tiempo asignado & $X$ \\
\hline & 21 & $\begin{array}{l}\text { Terminar las etapas educativas sin repetir ningún } \\
\text { curso }\end{array}$ & $x$ \\
\hline & 28 & $\begin{array}{l}\text { Conseguir notas altas para poder acceder a la } \\
\text { Universidad }\end{array}$ & $x$ \\
\hline & 42 & Esforzarme en tareas difíciles & $x$ \\
\hline & 43 & $\begin{array}{l}\text { Comparar los contenidos nuevos con los ya } \\
\text { adquiridos }\end{array}$ & $x$ \\
\hline & 47 & $\begin{array}{l}\text { Estudiar utilizando resúmenes, cuadros, } \\
\text { diagramas, etc. }\end{array}$ & $X$ \\
\hline & 54 & Planificar mis tareas y controlar su realización & $X$ \\
\hline & 57 & Obtener la aprobación de los profesores & $x$ \\
\hline Sociopolítica & 15 & Cambiar el sistema político actual & $x$ \\
\hline & 22 & Defender mis ideales políticos & $X$ \\
\hline & 36 & Pertenecer a un partido político & $\mathrm{X}$ \\
\hline & 38 & $\begin{array}{l}\text { Garantizar el derecho de autodeterminación de un } \\
\text { pueblo }\end{array}$ & $X$ \\
\hline & 46 & Participar en manifestaciones políticas & $x$ \\
\hline & 53 & $\begin{array}{l}\text { Apoyar a quienes utilizan la violencia en la } \\
\text { política }\end{array}$ & $X$ \\
\hline & 58 & Ser reconocido como un líder político & $X$ \\
\hline
\end{tabular}




\begin{tabular}{|c|c|c|c|c|}
\hline Compromiso & 70 & Conseguir las metas que me propongo & $\mathrm{X}$ & \\
\hline & 71 & Esforzarme poco en el logro de las metas & $x$ & \\
\hline & 72 & Esforzarme mucho en el logro de las metas & $\mathrm{X}$ & $x$ \\
\hline & 73 & Tomar las metas con cierta tranquilidad & $x$ & \\
\hline & 74 & $\begin{array}{l}\text { Cambiar de metas dependiendo de cómo vayan } \\
\text { las cosas }\end{array}$ & $X$ & \\
\hline & 75 & Evaluar las metas que consigo & $\mathrm{X}$ & $X$ \\
\hline & 76 & $\begin{array}{l}\text { Renunciar a las metas que requieren mucho } \\
\text { esfuerzo }\end{array}$ & $x$ & \\
\hline & 77 & $\begin{array}{l}\text { Estudiar la posibilidad de alcanzar determinadas } \\
\text { metas }\end{array}$ & $x$ & $x$ \\
\hline & 78 & Analizar el tiempo que tardaré en lograr una meta & $x$ & \\
\hline & 79 & $\begin{array}{l}\text { Trabajar con ilusión para conseguir las metas que } \\
\text { deseo }\end{array}$ & $x$ & $x$ \\
\hline
\end{tabular}

\section{Discusión y conclusiones}

El objetivo del presente estudio fue desarrollar una versión abreviada del Cuestionario de Metas para Adolescentes (CMA) (Sanz de Acedo Lizarraga et al., 2003) para su uso como instrumento de screening en la detección de metas adolescentes. Los resultados indicaron que el CMA, tanto en su versión original como en su versión reducida, presentó un buen comportamiento psicométrico. Además, la nueva versión corta desarrollada (CMA-R) resultó un autoinforme con garantías psicométricas para su utilización en la investigación.

Los niveles de consistencia interna en ambas versiones del CMA son similares a los encontrados en estudios previos (Sanz de Acedo Lizarraga et al., 2003). El análisis factorial confirmatorio realizado y los índices de discriminación permitieron seleccionar aquellos ítems que presentaron un mejor comportamiento psicométrico, dando lugar a una versión corta de 26 ítems con siete escalas; dichas escalas presentaron adecuados niveles de correlación con la versión original del CMA, indicativo de la validez de esta nueva versión reducida. Al ser este un trabajo que realiza una validación parsimoniosa del cuestionario original, podría esperarse que los resultados en lo referente a los índices fueran inferiores a lo deseable, pero la realidad es que se consigue mejorar la potencia estadística reduciendo en más de un $60 \%$ la longitud del cuestionario, manteniendo su estructura y funcionamiento original, ya que el análisis de la validez de constructo del CMA-R mostró, en consonancia con la literatura, que las metas presenta una estructura con 7 dimensiones (Sanz de Acedo Lizarraga et al., 2003).

En este sentido, las dimensiones del CMA engloban todos los niveles posibles de metas, no solo sociales o educativos, sino un espectro mucho más amplio y completo de la visión adolescente de su realidad. 
La utilización de autoinformes con un reducido número de ítems, como el CMA-R, para evaluación de las metas, permite una serie de ventajas respecto a otros métodos de evaluación, ya que es un método de rápida aplicación, y menos costoso de cara a su administración, puntuación e interpretación.

Los datos encontrados en este estudio deberían ser interpretados a la luz de algunas posibles limitaciones. En primer lugar, la adolescencia y la adultez temprana son etapas del desarrollo donde se producen cambios de tipo emocional y de formación de la identidad, que pueden limitar en el tiempo la interpretación de los datos obtenidos. En segundo lugar, en estudios donde se realiza una administración colectiva de cuestionarios suele presentarse una alta tasa de respuestas azarosas, por lo que también podría haber sido interesante la aplicación de alguna escala de infrecuencia de respuesta o de deseabilidad social, con la finalidad de detectar a los participantes con este tipo de perfil de respuestas. En tercer lugar, existe la limitación inherente a la aplicación de cualquier tipo de autoinforme, referida particularmente a la dificultad de algunos participantes para informar acerca de sus propios comportamientos, cogniciones y afectos y por último, en este artículo, el fin último ha sido la obtención de una versión abreviada del CMA basada en la aplicación de análisis factorial confirmatorio que atiende a criterios de validez interna, no obstante, esta validez es un aspecto que podría complementarse con elementos de validez externa, por lo tanto, este aspecto implica una limitación del estudio.

Resultaría interesante, de cara a futuras investigaciones, planteamientos que incluyan diferentes variables psicológicas, que supongan una mayor comprensión para el desarrollo de metas. Asimismo, sería interesante realizar estudios longitudinales con la finalidad de determinar la estabilidad de las metas encontradas, así como el rol que éstas puedan desempeñar a la hora de determinar la posible transición a la adultez.

\section{Referencias}

Armas, E. G., \& Hernández, H. M. (2006). ¿Por qué hacemos lo que hacemos?. Dimensiones básicas de la Motivación. Santa Cruz de Tenerife: Ediciones Idea.

Austin, J. T., \& Vancouver, J. B. (1996). Goal constructs in psychology: Structure, process, and content. Psychological Bulletin, 120(3), 338-375. doi: http://dx.doi.org/10.1037/00332909.120.3.338

Baker, D. A., \& Palmer, R. J. (2006). Examining the effects of perceptions of community and recreation participation on quality of life. Social Indicators Research, 75(3), 395418. doi: http://dx.doi.org/10.1007/s11205-004-5298-1

Berger, K. S. (2007). Psicología del desarrollo: infancia y adolescencia. Madrid: Editorial Médica Panamericana

Blackburn, R. (1993). The psychology of criminal conduct: Theory, research and practice. Nueva York, EEUU: Wiley.

Broc, M. A. (2000). Autoconcepto, autoestima y rendimiento académico en alumnos de $4^{\circ}$ de ESO Implicaciones psicopedagógicas en la orientación y tutoría. Revista de Investigación Educativa, 18(1), 119-146. Recuperado de http://revistas.um.es/rie/ article/view/121521/114201 
Brophy, J. (2005). Goal theorists should move on from performance goals. EducationalPsychologist, 40(3), 167-176. doi: http://dx.doi.org/10.1207/s15326985ep4003_3

Brown, A.T. (2006). El análisis factorial confirmatorio. Nueva York, EEUU: The Guilford Press.

Caprara, G. V., Barbaranelli, C., Pastorelli, C., \& Cervone, D. (2004). The contribution of self-efficacy beliefs to psychosocial outcomes in adolescence: predicting beyond global dispositional tendencies. Personality and Individual Differences, 37, 751-763. doi: http://dx.doi.org/10.1016/j.paid.2003.11.003

Carretero, M., Palacios, J., \& Marchesi, Á. (Comps.). (1985). Psicología evolutiva 3. Adolescencia, madurez y senectud. Madrid: Editorial Alianza.

Carroll, A., Durkin, K., Hattie, J., \& Houghton, S. (1997). Goal setting among adolescents: A comparison of delinquent, at-risk and not-at-riskyouth. Journal of EducationalPsychology, 89(3), 441-450. doi: http://dx.doi.org/10.1037/0022-0663.89.3.441

Casco, F. J., \& Oliva, A. (2005). Values and expectations on adolescence: discrepancies among parents, teachers, elders and adolescents. Infancia y Aprendizaje, 28(2), 209220. doi: http://dx.doi.org/10.1174/0210370053699302

Castillo, I., Balaguer, I., \& Duda, J. L. (2002). Goals perspectives of adolescents in sport context. Psicothema, 14(2), 280-287. Recuperado de http://dialnet.unirioja.es/ servlet/articulo?codigo $=1102276$

Cid, M. T., Espadalé, M. L., Carreras, F. B.,\& Martínez, L. M. (2013). Estudio sobre el desarrollo de la identidad en la adolescencia. Tendencias Pedagógicas, 21, 211-224.

Covington, M.V. (2000). Goal theory, motivation, and school achievement: An integrative review. Annual Reviews of Psychology, 51, 171-200. doi: http://dx.doi. org/10.1146/annurev.psych.51.1.171

Delgado, B., Inglés, C. J., García-Fernández, M., Castejón, J. L., \& Valle, A. (2010). Diferencias de género y curso en metas académicas en alumnos de Educación Secundaria Obligatoria. Revista Española de Pedagogía, 68(245), 67-83. Recuperado de http://www.jstor.org/stable/23766273

Domínguez, L. (2008). La adolescencia y la juventud como etapas del desarrollo de la personalidad. Distintas concepciones en torno a la determinación de sus límites y regularidades. Notas: Boletín Electrónico de Investigación de la Asociación Oaxaqueña de Psicología, 4(1), 69-76. Recuperado de http://www.conductitlan.net/ notas_boletin_investigacion/50_adolescencia_y_juventud.pdf

Duda, J. L., \& Nicholls, J. G. (1992). Dimensions of achievement motivation in schoolwork and sport. Journal of Educational Psychology, 84(3), 290-299. doi: http://dx.doi. org/10.1037/0022-0663.84.3.290

Dweck, C. S. (1986). Motivational processes affecting learning. The American Psychologist, 41(10), 1040-1048. doi: http://dx.doi.org/10.1037/0003-066X.41.10.1040

Elkind, D. (1967). Egocentrism in adolescence. Child Development, 38(4), 1025-1034.

Elliot, A. J., Gable, S. L., \& Mapes, R. R. (2006). Approach and avoidance motivation in the social domain. Personality and Social Psychology Bulletin, 32(3), 378-391. doi: http://dx.doi.org/10.1177/0146167205282153

Emler, N., \& Reicher, S. (1995). La adolescencia y la delincuencia: La gestión colectiva de reputación. Oxford, Reino Unido: Blackwell Publishing.

Erikson, E. H. (1970). Infancia y sociedad. Buenos Aires, Argentina: Editorial Hormé. 
Ford, M. E., \& Nichols, C. W. (1992). Assessment of personal goals. Palo Alto, EEUU: Consulting Psychologist Press.

Freitas-Rosa, M., Gonçalves, S., \& Antunes, H. (2015). Prevalencia y factores de predicción de los consumos de alcohol y tabaco en la adolescencia: El papel de la condición de pesos, de la condición clínica y de las dimensiones psicosociales. Anales de Psicología, 31(1), 217-225. doi: http://dx.doi.org/10.6018/analesps.31.1.158671

Gardner, M., Roth, J., \& Brooks-Gunn, J. (2008). Adolescents' participation in organized activities and developmental success 2 and 8 years after high school: do sponsorship, duration, and intensity matter?. Developmental Psychology, 44(3), 814-830. doi: http://dx.doi.org/10.1037/0012-1649.44.3.814.

Gaxiola, J., González, S., Contreras, Z., \& Gaxiola, E. (2012). Predictores del rendimiento académico en adolescentes con disposiciones resilientes y no resilientes. Revista de Psicología, 30(1), 47-74.

Gibson-Cline, J. (Ed.). (2000). Youth and coping in Twelve Nations: Surveys of 18-20-yearold young people. Nueva York, EEUU: Routledge.

González, M. L. G., \& Hernández, P. M. (2009). Estrés y adolescencia: estrategias de afrontamiento y autorregulación en el contexto escolar. Studium: Revista de Humanidades, 15, 327-344. Recuperado de http://dialnet.unirioja.es/descarga/articulo/3074506.pdf

González, J., \& Portolés, A. (2014). Actividad física extraescolar: relaciones con la motivación educativa, rendimiento académico y conductas asociadas a la salud. RIPED. Revista Iberoamericana de Psicología del Ejercicio y el Deporte, 9(1), 51-65.

Grotevant, H. D. (1998). Adolescent development in family contexts. En W. Damon \& N. Eisenberg (Eds.), Handbook of Child Psychology. (Vol. 3: Social, Emotional, and Personality Development, Ed. 5, pp. 1097-1149). Nueva York, EEUU: Wiley.

Guan, J., Xiang, P., McBride, R., \& Bruene, A. (2006). Achievement goals, social goals, and students' reported persistence and effort in high school physical education. Journal of Teaching in Physical Education, 25(1), 58-74. doi: http://dx.doi.org/10.1123/ jtpe.25.1.58

Hechinger, F. M. (1992). Fateful Choices: Healthy Youth for the 21st Century. Nueva York, EEUU: Hill and Wang Publishers.

Inglés, C. J., Martínez-Monteagudo, M. C., García-Fernández, J. M., Valle, A., \& Castejón, J. L. (2015). Perfiles de orientación de meta y el autoconcepto de Estudiantes Secundarios. Psicodidáctica, 20(1), 99-116. Recuperado de http://www.ehu.eus/ojs/ index.php/psicodidactica/article/view/10023/10396

Kinnebrew, J. S., Biswas, G., Sulcer, B., \& Taylor, R. S. (2013). Investigating selfregulated learning in teachable agent environments. En R. Azevedo \& V. Aleven (Eds.), International Handbook of Metacognition and Learning Technologies. (Vol. 26, pp. 451-470). Nueva York, EEUU: Springer.

Kline, R. B. (2005). Principies and practice of structural equation modeling (Ed. 2). Nueva York, EEUU: Guilford.

Krauskopf, D. (1982). Adolescencia y educación. Chile: Editorial Universidad Estatal a Distancia.

Kroger, J. (2004). Identity in adolescence: The balance between self and other. Nueva York, EEUU: Psychology Press. 
Liau, A. K. (2007). Promoting children's personal strengths: Positive psychology goes to school. En A. G. Tan (Ed.), Creativity: A handbook for teachers (pp. 441-466). Singapore: World Scientific.

Markus, H., \& Nurius, P. (1986). Possible selves. American psychologist, 41(9), 954-969. doi: http://dx.doi.org/10.1037/0003-066X.41.9.954

Markus, H., \& Kunda, Z. (1986). Stability and malleability of the self-concept. Journal of Personality and Social Psychology, 51(4), 858-866.

Martínez, S. R., Cabanach, R. G., \& Pérez, J. C. N. (2004). Diferencias en el uso de self-handicapping y pesimismo defensivo y sus relaciones con las metas de logro, la autoestima y las estrategias de autorregulación. Psicothema, 16(4), 625-631.

Mayseless, O., \& Scharf, M. (2007). Adolescents' attachment representations and their capacity for intimacy in close relationships. Journal of Research on Adolescence, 17(1), 23-50. doi: http://dx.doi.org/10.1111/j.1532-7795.2007.00511.x

Molina, J., Chorot, P., Valiente, R. M., \& Sandín, B. (2014). Miedo a la evaluación negativa, autoestima y presión psicológica: Efectos sobre el rendimiento deportivo en adolescentes. Cuadernos de Psicología del Deporte, 14(3), 57-66. doi: http://dx.doi. org/10.4321/S1578-84232014000100006

Monereo, C. (2007). Hacia un nuevo paradigma del aprendizaje estratégico: el papel de la mediación social, del self y de las emociones. Revista de Investigación Educativa, 5(3), 239-265.

Navas, L., Soriano, J. A., Holgado, F. P. L., \& López, M. (2009). Las orientaciones de meta de los estudiantes y los deportistas: Perfiles motivacionales. Acción Psicológica, 6(2), 17-29.

Nicholls, J. G. (1989). The competitive ethos and democratic education. Cambridge, EEUU: Harvard University Press.

Paoloni, P. V., Rinaudo, M. C., \& González-Fernández, A. (2011). Procesos de retroalimentación en la autorregulación de recursos de aprendizaje. Explorando su potencial en el contexto de la universidad. Revista de Educación a Distancia. Sección Docencia Universitaria en la Sociedad del Conocimiento, 3, 1-18. Recuperado de https:// www.um.es/ead/reddusc/3/paoloni_et_al.pdf

Pintrich, P. R., \& Schunk, D. H. (2006). Motivación en contextos educativos. Teoría, investigación y aplicaciones (2a. ed.). Madrid: Pearson Educación.

Portolés, A. (2013). Orientación de metas y práctica de actividad física en adolescentes. Implicaciones sobre el rendimiento académico y el burnout. (Tesis doctoral, Universidad de Murcia). Recuperada de https://digitum.um.es/xmlui/handle/10201/39908

Ramos, F. J. C., \& Delgado, A. O. (2004). Ideas sobre la adolescencia entre padres, profesores, adolescentes y personas mayores. Apuntes de Psicología, 22(2), 171185. Recuperado de http://www.apuntesdepsicologia.es/index.php/revista/article/ view/49

Ratcliff, M. B., Jenkins, T. M., Reiter-Purtill, J., Noll, J. G., \& Zeller, M. H. (2011). La asunción de riesgos comportamientos de los adolescentes con obesidad extrema: ¿normativa o no?. Pediatría, 127(5), 827-834.

Rijo, A. G., Moreno, J. H., Herráez, I. M., \& Medina, S. G. (2014). Necesidades psicológicas básicas en Educación Física según el género y el curso del estudiante. 
Revista de Investigación Educativa, 32(1), 159-167. doi: http://dx.doi.org/10.6018/ rie.32.1.172311

Riveiro, J. M. S., Sánchez, V. R., Horcajo, R. A., \& Suárez, A. P. F. (2013). Metas y Compromiso de los opositores al cuerpo de maestros en la especialidad de Educación Primaria. Revista de Investigación Educativa, 31(1), 77-92. doi: http://dx.doi. org/10.6018/rie.31.1.139661

Romero, J. C. G., Lugo, S. G., \& Villa, E. G. (2013). Autorregulación, Resiliencia y Metas Educativas: Variables Protectoras del Rendimiento Académico de Bachilleres. Revista Colombiana de Psicología, 22(2), 241-252.

Rueda, P. O. (2014). Competencias básicas y procesos perceptivos: factores claves en la formación y orientación de los jóvenes en riesgo de exclusión educativa y sociolaboral. Revista de Investigación Educativa, 32(2), 531-546. doi: http://dx.doi. org/10.6018/rie.32.2.181551

Salmerón, H., Gutierrez-Braojos, C., Salmerón-Vilchez, P., \& Rodríguez, S. (2011). Metas de logro, estrategias de regulación y rendimiento académico en diferentes estudios universitarios. Revista de Investigación Educativa, 29(2), 467-486. Recuperado de http://revistas.um.es/rie/article/view/116041

Santillano, I. (2009). La adolescencia: añejos debates y contemporáneas realidades. Última Década, 17(31), 55-71. doi: http://dx.doi.org/10.4067/S0718-22362009000200004

Sanz de Acedo Lizarraga, M., Ugarte, M. D., \& Lumbreras, M.V. (2003). Desarrollo y validación de un Cuestionario de Metas para Adolescentes. Psicothema, 15(3), 493-499. Recuperado de http://www.unioviedo.net/reunido/index.php/PST/article/ view/8100

Sanz de Acedo Lizarraga, M., Ugarte, M. D., Lumbreras, M. V., \& Sanz de Acedo Baquedano, M. (2006). Goals and personality in adolescents. School Psychology International, 27(3), 370-381.

Suárez, L. A., Delgado, A. O., \& Jiménez, A. M. L. (2011). Desarrollo y validación de una escala de valores para el desarrollo positivo adolescente. Psicothema, 23(1), 153-159. Recuperado de http://www.psicothema.com/pdf/3864.pdf

Valle, A., Núñez Pérez, J. C., Cabanach, R. G., Rodríguez, S., González-Pienda, J. A., \& Rosario, P. (2007). Metas académicas y estrategias motivacionales de autoprotección. Revista Electrónica de Investigación Psicoeducativa, 5(13), 617-632. Recuperado de http://repositorium.sdum.uminho.pt/handle/1822/11924

Viñas, F., González, M., García, Y., Malo, S., \& Casas, F. (2015). Los estilos y estrategias de afrontamiento y su relación con el bienestar personal en una muestra de adolescentes. Anales de Psicología, 31(1), 226-233. doi: http://dx.doi.org/0.6018/ analesps.31.1.163681

Waterman, A. S. (1999). Identity, the identity statuses, and identity status development: A contemporary statement. Developmental Review, 19(4), 591-621. Recuperado de http://www.sciencedirect.com/science/article/pii/S0273229799904938

Wentzel, K. R. (1993). Motivation and achievement in early adolescence. The Role of multiple classroom goals. The Journal of Early Adolescence, 13(1), 4-20. doi: http:// dx.doi.org/10.1177/0272431693013001001 
Wentzel, K. R., \&Wigfield, A. (1998). Academic and social motivational influences on students' academic performance. Educational Psychology Review, 10(2), 155-175. doi: 10.1023/A:1022137619834

Zacarés, J., Iborra, A., Tomás, J., \& Serra, E. (2009). El desarrollo de la identidad en la adolescencia y adultez emergente: Una comparación de la identidad global frente a la identidad en dominios específicos. Anales de Psicología, 25(2), 316-329.

Zimmerman, B. J. (2008). Investigating Self-Regulation and Motivation: Historical Background, Methodological Developments, and Future Prospects. American Educational Research Journal, 45(1), 166-183. doi: http://dx.doi.org/10.3102/0002831207312909

Fecha de recepción: 06 de mayo de 2015

Fecha de revisión: 06 de mayo de 2015

Fecha de aceptación: 24 de octubre de 2016 